\title{
Models of Finseler Spaces With Given Geodesics
}

\author{
K.B. Pandey , B. K. Tripathi \& R.B. Tiwari \\ Department of mathematics, K.N.I.T. Sultanpur (U.P.) 228118,INDIA, \\ Department of mathematics (Ministry of Chemical \& Fertilizers, \\ Govt. of India), Lucknow, U.P.-226008
}

Abstract: In the present paper, we introduce the theory of four dimensional Finsler space and define geodesic equation with the basis of Finsler space. We also try to define geodesic equation to useful significance.

\section{Introduction}

Finseler geometry is a kind of differential geometry, which was originated by P. Finselerin 1918. It is usually considered as a generalization of Riemannian geometry. The definition of finseler space-

\subsection{Finseler Space:}

Suppose that we are given a function $\mathrm{L}\left(x^{i}, y^{i}\right)$ of the line element $\left(x^{i}, y^{i}\right)$ of a curve defined in R. We shall assume $\mathrm{L}$ as a function of class at least $\mathrm{C}^{5}$ in all its $2 \mathrm{n}$-arguments. If we define the infinitesimal distance $\mathrm{ds}$ between two points $P\left(x^{i}\right)$ and $\mathrm{Q}\left(x^{i}+d x^{i}\right)$ of $\mathrm{R}$ by the relation

$$
d s=L\left(x^{i}, d x^{i}\right)
$$

then the manifold $M^{n}$ requipped with the fundamental function $\mathrm{L}$ defining the metrix (1.1.1) is called a Finsler space if $\mathrm{L}\left(x^{i}, d x^{i}\right)$ satisfies the following conditions.

\section{Condition A-}

The function $\mathrm{L}\left(x^{i}, y^{i}\right)$ is positively homogeneous of degree one in $y^{i}$ i.e.

Condition B-

$$
\mathrm{L}\left(x^{i}, k y^{i}\right)=k L\left(x^{i}, y^{i}\right), \mathrm{k}>0
$$

The function $\mathrm{L}\left(x^{i}, y^{i}\right)$ is positively if not all $y^{i}$ vanish simultaneously i.e.

\section{Condition C-}

$$
\mathrm{L}\left(x^{i}, y^{i}\right)>0 \text { with } \sum_{i}\left(y^{i}\right)^{2} \neq 0
$$

The quadratic form

$$
\dot{\partial}_{j} L^{2}(x, y) \varepsilon^{i} \varepsilon^{j}=\frac{\partial^{2} L^{2}(x, y)}{\partial y^{i} \partial y^{j}} \varepsilon^{i} \varepsilon^{j}
$$

is assumed to be positive definite for any variable $\varepsilon^{i}$.

Form Euler's theorem on homogenous functions, we have

$$
\begin{aligned}
& \dot{\partial}_{i} L(x, y) \dot{y}^{i}=L(x, y) \\
& \dot{\partial}_{i} \dot{\partial}_{j} L^{2}(x, y) y^{i}=0
\end{aligned}
$$

We put

$$
g_{i j}(x, y)=\frac{1}{2} \dot{\partial}_{i} \dot{\partial}_{j} L^{2}(x, y)
$$

Using the theory of quadratic form and the condition $C$, we deduce form (1.1.4) that-

$$
g(x, y)=g_{i j}(x, y)>0
$$

for all line elements $\left(x^{i}, y^{i}\right)$. If the function $\mathrm{L}$ is of particular form

$$
L\left(x^{i}, d x^{i}\right)=\left[g_{i j}\left(x^{k}\right) d x^{i} d x^{j}\right]^{1 / 2}
$$

where the coefficients $g_{i j}\left(x^{k}\right)$ are independent of $d x^{i}$, the metric defined by this function is called Riemannian metric and manifold $M^{n}$ is called a Riemannian space. Throughout the paper, $F^{n}$ or $\left(M^{n}, L\right)$ will denote the $\mathrm{n}$-dimensional finsler space, where as n-dimensional Riemannian space will be denoted by $R^{n}$.

\subsection{Intrinsic Fields of Orthonormal Frames :}

Berwald theory of two-dimensional Finsler space is developed based on the intrinsic field of orthonormal frame which consists of the normalized supporting element $l^{i}$ and unit vector orthonormal to $l^{i}$. Following idea Moor introduced, in a three-dimensional Finsler space, the intrinsic field of orthonormal frame which consists of the normalized supporting element $l^{i}$, the normalized torsion vector $C^{i} / C$ and a unit vector orthogonal to them and developed a theory of three-dimensional Finsler spaces. Generalizing the Berwald's and Moor's ideas, Miron and Matsumoto[ (1986), (1977), (1989)] developed a theory of intrinsic orthonormal frame fields on $\mathrm{n}$-dimensional Finsler space as follows. 
Let $L(x, y)$ be the fundamental function of an n-dimensional Finsler space and introduce Finsler tensor fields of $(0,2 \alpha-1)$ type, $\alpha=1,2, \ldots ., \mathrm{n}$ by

$$
L_{i_{1} i_{2} \ldots . . i_{2 \alpha-1}}=\frac{1}{2^{\alpha}} \dot{\partial}_{i_{1}} \dot{\partial}_{i_{2}} \ldots \ldots . \dot{\partial}_{i_{2 \alpha-1}} L^{2}
$$

Then we get a sequence of covariant vectors

$$
L_{\alpha) i}=L_{i j_{1} j_{2} \ldots . . j_{2 \alpha-3} \cdot j_{2 \alpha-2}} g^{j_{1}} g^{j_{1}} \ldots \ldots . g^{j_{2 \alpha-3} \cdot j_{2 \alpha-2}}
$$

Definition-1: If (n-1) covariant vectors $L_{\alpha) i}, \alpha=1,2, \ldots ., \mathrm{n}-1$ are linearly independent, the Finsler space is called strongly non-Riemannian.

Assuming above n-covectors $L_{\alpha) i}$ are linearly independent and put $e_{1)}^{i}=L_{1)}^{i} / L=l^{i}$. Here and in following we use raising and lowering of indices as $L_{1)}^{i}=g^{i j} L_{1) j}$.

Further putting $N_{1) i j}=g_{i j}-e_{1) i} e_{1) j}$ and matrix $N_{1)}=N_{1) i j}$ is of rank (n-1). Second vector $\boldsymbol{e}_{2}$ ) is introduced by $e_{2)}^{i}=L_{2)}^{i} / L_{2}$,

where, $L_{2}$ is the length of $L_{2)}^{i}$ relative to $y^{i}$. Next we put $N_{2) i j}=N_{1) i j}-e_{2) i} e_{2) j}, E_{3)}^{i}=N_{2) j}^{i} L_{3)}^{j}$ and so third vector $e_{3)}$ is defined by,

$e_{3)}^{i}=E_{3)}^{i} / E_{3}$,

where, $E_{2}$ is the length of $E_{2}^{i}$ relative to $\boldsymbol{y}^{i}$. The repetition of above process gives a

vector $e_{r+1)}, r=1,2, \ldots \ldots, \mathrm{n}-1$ defined by

$$
e_{r+1)}^{i}=E_{r+1)}^{i} / E_{r+1}
$$

where, $E_{r+1)}^{i}=N_{r) j}^{i} L_{r+1)}^{j} E_{r+1}$ is the length of $E_{r+1)}^{i}$ relative to $y^{i}$ and $N_{r) i j}=N_{r-1) i j}-e_{r) i} e_{2) j}$.

Definition-2: The orthonormal frame $\left\{e_{\alpha}\right\}, \alpha=1,2, \ldots, n$ as above defined in every in every co-ordinate neighborhood of a strongly non-Riemannian Finsler space is called the 'Miron Frame'.

Consider the Miron frame $\left\{e_{\alpha}\right\}$, If a tensor $T_{j}^{i}$ of $(1,1)$-type, for instance, is given then $T_{j}^{i}=T_{\alpha \beta} e_{\alpha)}^{i} e_{\beta) j}$

where, the scalars $T_{\alpha \beta}$ are defined as

$$
T_{\alpha \beta}=T_{j}^{i} e_{\alpha) i} e_{\alpha)}^{j}
$$

These scalars $T_{\alpha \beta}$ are called the scalar components of $T_{j}^{i}$ with respect to Miron frame.

Let $H_{\alpha) \beta \gamma}$ be scalar components of the h-covariant derivatives $\boldsymbol{e}_{\boldsymbol{\alpha}) \mid \boldsymbol{j}}^{\boldsymbol{i}}$ and $V_{\alpha) \beta \gamma} / L$ be scalar components of the vcovariant derivatives $e_{\alpha)}^{i} l_{j}$ with respect to $\mathrm{C} \Gamma$ of the vector $e_{\alpha)}^{i}$ belonging to the Miron frame. Then

$e_{\alpha) \mid j}^{i}=H_{\alpha) \beta \gamma} e_{\beta)}^{i} e_{\gamma) j}$,

$\left.e_{\alpha)}^{i}\right|_{j}=V_{\alpha) \beta \gamma} e_{\beta)}^{i} e_{\gamma) j}$,

where, the scalars $H_{\alpha) \beta \gamma}$ and $V_{\alpha) \beta \gamma}$ satisfying the following relations [Berwald (1947)].

$$
H_{1) \beta \gamma}=0, H_{\alpha) \beta \gamma}=-H_{\beta) \alpha \gamma} \text {, }
$$

$V_{\alpha) \beta \gamma}=\delta_{\beta \gamma}-\delta_{\beta}^{1} \delta_{\gamma}^{1}, \quad V_{\alpha) \beta \gamma}=-V_{\beta) \alpha \gamma}$

Definition-3: The scalars $H_{\alpha) \beta \gamma}$ and $V_{\alpha) \beta \gamma}$ are called connection scalars.

If $C_{\alpha \beta \gamma} / L$ be the scalar components of the (h)hv-torsion tensor $C_{j k}^{i}$ i.e.,

then [Hambo,H(1934)], we have

$$
L C_{j k}^{i}=C_{\alpha \beta \gamma} e_{\alpha)}^{i} e_{\beta) j} e_{\gamma) k}
$$

\section{Proposition-1:}

i. $\quad C_{1 \beta \gamma}=0$

ii. $\quad C_{2 \mu \mu}=L C, C_{3 \mu \mu}=\cdots \ldots \ldots=C_{n \mu \mu}=0$ for $n \geq 3$, where $C$ is the length of $C^{i}$.

Now, we consider scalar components of covariant derivatives of a tensor field, for instance, $T_{j}^{i}$. Let $T_{\alpha \beta, \gamma}$ and $T_{\alpha \beta ; \gamma} / L$ be the scalar components of h-and v-covariant derivatives with respect to $\mathrm{C} \Gamma$ respectively of a tensor $T_{j}^{i}$ i.e.,

$$
\begin{gathered}
T_{j \mid k}^{i}=T_{\alpha \beta, \gamma} e_{\alpha)}^{i} e_{\beta) j} e_{\gamma) k} \\
\text { and } \\
\left.L T_{j}^{i}\right|_{k}=T_{\alpha \beta ; \gamma} e_{\alpha)}^{i} e_{\beta) j} e_{\gamma) k},
\end{gathered}
$$

then we have [Hambo,H(1934)],

$$
\begin{aligned}
& T_{\alpha \beta, \gamma}=\left(\delta_{k} T_{\alpha \beta}\right) e_{\gamma)}^{k}+T_{\mu \beta} H_{\mu) \alpha \gamma}+T_{\alpha \mu} H_{\mu) \beta \gamma} \\
& \text { and } T_{\alpha \beta ; \gamma}=L\left(\dot{\partial}_{k} T_{\alpha \beta}\right) e_{\gamma)}^{k}+T_{\mu \beta} V_{\mu) \alpha \gamma}+T_{\alpha \mu} V_{\mu) \beta \gamma} .
\end{aligned}
$$

The scalar components $T_{\alpha \beta, \gamma}$ and $T_{\alpha \beta ; \gamma}$ are called h-and v-scalar derivative of $T_{\alpha \beta}$ respectively. 


\section{(i) Two-dimensional Finsler space}

The Miron frame $\left\{e_{1)}, e_{2}\right\}$ is called the Berwald frame. The first vector $e_{1 \text { ) }}^{i}$ is the normalized supporting element $l^{i}=y^{i} / L$ and the second vector $e_{2)}^{i}=m^{i}$ is the unit vector orthogonal to $l^{i}$. If $C^{i}$ has nonzero length $C$, them ${ }^{i}= \pm C^{i} / C$. The connection scalars $H_{\alpha) \beta \gamma}$ and $V_{\alpha) \beta \gamma}$ of a two-dimensional Finsler space are such that

$$
\begin{aligned}
& H_{\alpha) \beta \gamma}=0, \quad V_{\alpha) \beta 1}=0, \quad V_{\alpha) \beta 2}=\delta_{\alpha \beta}^{12}, \text { which implies } \\
& l_{\mid j}^{i}=0, \quad m_{\mid j}^{i}=0,\left.L l^{i}\right|_{j}=m^{i} m_{j},\left.\quad L m^{i}\right|_{j}=-l^{i} m_{j}(1.2 .5)
\end{aligned}
$$

There is only one surviving scalar components of $L C_{i j k}$ namely $C_{222}$. If we put $I=C_{222}$.

Then $\quad L C_{i j k}=I m_{i} m_{j} m_{k}$

The scalar $l$ is called the main scalar of a two-dimensional Finsler space.

Proposition-2: In a two-dimensional Finsler space

i. The h-curvature tensor $R_{h i j k}$ of $\mathrm{C} \Gamma$ is written as,

ii. The hv-curvature tensor $P_{h i j k}$ of $\mathrm{C} \Gamma$ is written as,

$$
R_{h i j k}=R\left(l_{h} m_{i}-l_{i} m_{h}\right)\left(l_{j} m_{k}-l_{k} m_{j}\right)
$$

$P_{h i j k}=I_{, 1}\left(l_{h} m_{i}-l_{i} m_{h}\right) m_{j} m_{k}$

iii. The (v)hv-curvature tensor $P_{i j k}$ is written as,

$P_{i j k}=I_{, 1} m_{i} m_{j} m_{k}$

\section{(ii) Three-dimensional Finsler space}

The Miron frame of a three-dimensional Finsler space is called the Moor-frame. The first vector $e_{1 \text { ) }}^{i}$ of Moor-frame $\left\{e_{1)}, e_{2)}, e_{3)}\right\}$ is the normalized supporting element $\boldsymbol{l}^{i}$, the second vector $e_{2 \text { ) }}^{i}$ is the normalized torsion vector $m^{i}=C^{i} / C$ and the thirde $e_{3)}^{i}=n^{i}$ is constructed by,

$$
n^{i}=\varepsilon^{i j k} e_{1) j} e_{2) k} \text { where, } \varepsilon^{i j k}=g^{-(1 / 2)} \delta_{123}^{i j k}
$$

Now, following two Finsler vector fields are defined [Mastsumoto(1986)]

$\mathrm{h}_{i}=\mathrm{h}_{\gamma} e_{\gamma) i}$ and $v_{i}=v_{\gamma} e_{\gamma) i}$ then we have,

$$
\begin{aligned}
H_{\alpha) \beta \gamma}= & {\left[\begin{array}{ccc}
0 & 0 & 0 \\
0 & 0 & \mathrm{~h}_{\gamma} \\
0 & -\mathrm{h}_{\gamma} & 0
\end{array}\right], \quad V_{\alpha) \beta \gamma}=\left[\begin{array}{ccc}
0 & \delta_{\gamma}^{2} & \delta_{\gamma}^{3} \\
-\delta_{\gamma}^{2} & 0 & v_{\gamma} \\
-\delta_{\gamma}^{3} & -v_{\gamma} & 0
\end{array}\right] } \\
& \left\{\begin{array}{l}
l_{\mid j}^{i}=0 \\
m_{\mid j}^{i}=\left.\left.n^{i}\right|_{j} \mathrm{~h}_{\mathrm{j}} L m^{i}\right|_{j}=-l^{i} m_{j}+n^{i} v_{j} \\
n_{\mid j}^{i}=-\left.m^{i} \mathrm{~h}_{j} L n^{i}\right|_{j}=-l^{i} n_{j}-m^{i} v_{j}
\end{array}\right.
\end{aligned}
$$

Definition-4: The Finsler vector fields $\mathrm{h}_{i}$ and $v_{i}$ defined in (2.2.6) are called the $\mathrm{h}$-and v-connection vectors of a three-dimensional Finsler space.

The (h)hv-torsion tensor of a three-dimensional Finsler space is given by [Mastusmoto(1986)],

$L C_{i j k}=H m_{i} m_{j} m_{k}-J \pi_{(i j k)}\left(m_{i} m_{j} n_{k}\right)+I \pi_{(i j k)}\left(m_{i} n_{j} n_{k}\right)+J \mathrm{n}_{\mathrm{i}} \mathrm{n}_{\mathrm{j}} \mathrm{n}_{\mathrm{k}}$

The three scalar fields $H$, Iand $J$ of (1.2.8) are called the main scalars of a three-dimensional Finsler space and $\pi_{(\mathrm{ijk})}$ represent cyclic sum of the terms obtained by cyclic permutation of $i, j, k$.

The h-and v-connection vectors of a three-dimensional space has been firstly solved, in terms of main scalars explicitly, by Ikeda (1994).

\section{(iii) Four-dimensional Finslar space}

Prof. T. N. Pandey and D. K. Dwevidi developed the theory of four-dimensional Finsler spaces in the year 1997 in terms of scalars, taking $\mathrm{l}^{\mathrm{i}}, \mathrm{m}^{\mathrm{i}}, \mathrm{n}^{\mathrm{i}}$ and a unit vector $\mathrm{p}^{\mathrm{i}}$ perpendicular to $\mathrm{l}^{\mathrm{i}}, \mathrm{m}^{\mathrm{i}}, \mathrm{n}^{\mathrm{i}}$ as $\mathrm{p}^{\mathrm{i}}=\epsilon^{\mathrm{ijkl}} \mathrm{l}_{\mathrm{j}} \mathrm{m}_{\mathrm{k}} \mathrm{n}_{\mathrm{l}}$. The orthonormal frame $\left(\mathrm{l}^{\mathrm{i}}, \mathrm{m}^{\mathrm{i}}, \mathrm{n}^{\mathrm{i}}, \mathrm{p}^{\mathrm{i}}\right)$ as above defined in every coordinate neighborhood of a strongly nonRiemannian Finsler space is called Miron frame.

M. Matsumoto defines the scalar component of a tensor in Miron frame as follows:-

If a tensor $\mathrm{T}_{\mathrm{jk}}^{\mathrm{i}}$ of $(1,2)$ type for instance is given, we define scalars

Then $\mathrm{T}_{\mathrm{jk}}^{\mathrm{i}}$ is written in the form,

$$
\mathrm{T}_{\alpha \beta \gamma}=\mathrm{T}_{\mathrm{jk}}^{\mathrm{i}} \mathrm{e}_{\alpha) \mathrm{i}} \mathrm{e}_{\beta)}^{\mathrm{j}} \mathrm{e}_{\gamma)}^{\mathrm{k}}
$$

$$
\mathrm{T}_{\mathrm{jk}}^{\mathrm{i}}=\mathrm{T}_{\alpha \beta \gamma} \mathrm{e}_{\alpha)}^{\mathrm{i}} \mathrm{e}_{\beta) \mathrm{j}} \mathrm{e}_{\gamma) \mathrm{k}}
$$


These $\mathbf{T}_{\alpha \beta \gamma}$ are called scalar components of $\mathbf{T}_{\mathbf{j} \mathbf{k}}^{\mathbf{i}}$ with respect to Miron frame

From the equations

$$
\mathrm{e}_{1)}^{\mathrm{i}}=\mathrm{l}^{\mathrm{i}}, \quad \mathrm{e}_{2)}^{\mathrm{i}}=\mathrm{m}^{\mathrm{i}}, \quad \mathrm{e}_{3)}^{\mathrm{i}}=\mathrm{n}^{\mathrm{i}}, \quad \mathrm{e}_{4}^{\mathrm{i}}=\mathrm{p}^{\mathrm{i}} .
$$

and

$$
g_{i j} j^{i} l^{j}=g_{i j} m^{i} m^{j}=g_{i j} n^{i} n^{j}=g_{i j} p^{i} p^{j}=1
$$

we have,

$$
g_{i j}{ }^{i} m^{j}=g_{i j}{ }^{i} n^{j}=g_{i j} l^{i} p^{j}=g_{i j} m^{i} n^{j}=g_{i j} m^{i} p^{j}=g_{i j} n^{i} p^{j}=0
$$

$$
\mathrm{g}_{\mathrm{ij}}=\mathrm{l}_{\mathrm{i}} \mathrm{l}_{\mathrm{j}}+\mathrm{m}_{\mathrm{i}} \mathrm{m}_{\mathrm{j}}+\mathrm{n}_{\mathrm{i}} \mathrm{n}_{\mathrm{j}}+\mathrm{p}_{\mathrm{i}} \mathrm{p}_{\mathrm{j}}
$$

Next, the $\mathrm{C}_{\text {-tensor }} \mathrm{C}_{\mathrm{ijk}}=\frac{1}{2} \dot{\partial}_{\mathrm{k}} \mathrm{g}_{\mathrm{ij}}$ satisfies $\mathrm{C}_{\mathrm{ijk}} \mathrm{l}^{\mathrm{i}}=\mathrm{C}_{\mathrm{ijk}} \mathrm{j}^{\mathrm{j}}=\mathrm{C}_{\mathrm{ijk}} \mathrm{l}^{\mathrm{k}}=0$. So we have the expression of $\mathrm{C}_{\mathrm{ijk}}$ in the form

$$
\begin{aligned}
\mathrm{LC}_{\mathrm{ijk}}= & H m_{\mathrm{i}} \mathrm{m}_{\mathrm{j}} \mathrm{m}_{\mathrm{k}}+\mathrm{Jn}_{\mathrm{i}} \mathrm{n}_{\mathrm{j}} \mathrm{n}_{\mathrm{k}}+\mathrm{H}^{\prime} \mathrm{p}_{\mathrm{i}} \mathrm{p}_{\mathrm{j}} \mathrm{p}_{\mathrm{k}}+\mathrm{I} \pi_{(\mathrm{ijk})}\left\{\mathrm{m}_{\mathrm{i}} \mathrm{n}_{\mathrm{j}} \mathrm{n}_{\mathrm{k}}\right\} \\
& \quad+\mathrm{K} \pi_{(\mathrm{ijk})}\left\{\mathrm{m}_{\mathrm{i}} \mathrm{p}_{\mathrm{j}} \mathrm{p}_{\mathrm{k}}\right\}+\mathrm{J}^{\prime} \pi_{(\mathrm{ijk} \mathrm{k})}\left\{\mathrm{n}_{\mathrm{i}} \mathrm{p}_{\mathrm{j}} \mathrm{p}_{\mathrm{k}}\right\}-\left(\mathrm{J}+\mathrm{J}^{\prime}\right) \pi_{(\mathrm{ijk})}\left\{\mathrm{n}_{\mathrm{i}} \mathrm{m}_{\mathrm{j}} \mathrm{m}_{\mathrm{k}}\right\} \\
& +\mathrm{I}^{\prime} \pi_{(\mathrm{ijk})}\left\{\mathrm{n}_{\mathrm{i}} \mathrm{n}_{\mathrm{j}} \mathrm{p}_{\mathrm{k}}\right\}-\left(\mathrm{H}^{\prime}+\mathrm{I}^{\prime}\right) \pi_{(\mathrm{ijk})}\left\{\mathrm{m}_{\mathrm{i}} \mathrm{m}_{\mathrm{j}} \mathrm{p}_{\mathrm{k}}\right\}+\mathrm{K}^{\prime} \pi_{(\mathrm{ijk})}\left\{\mathrm{m}_{\mathrm{i}} \mathrm{n}_{\mathrm{j}} \mathrm{p}_{\mathrm{k}}\right\}
\end{aligned}
$$

where, $H, I, J, K, \mathrm{H}^{\prime}, \mathrm{I}^{\prime}, \mathrm{J}^{\prime}, \mathrm{K}^{\prime}$ are called main scalars satisfying $H+I+K=L C$.

Now we denote the $h$-and v-covariant differentiations of a tensor field with respect to

$\mathbf{C}^{\mathbf{i}}$ by the short line $\left(_{(i)}\right)$ and long line (ii) respectively, the following equations are derived

$$
\begin{gathered}
\left\{\begin{array}{c}
L_{\mid i}=0, \quad l_{i \mid j}=0, \quad m_{i \mid j}=n_{i} h_{j}-p_{i} j_{j}, \\
n_{i \mid j}=p_{i} k_{j}-m_{i} h_{j}, \quad p_{i} \mid j=m_{i} j_{j}-n_{i} k_{j}
\end{array}\right. \\
L_{i}\left|j=h_{i j}, \quad L m_{i}\right|_{j}=-l_{i} m_{j}+n_{i} u_{j}+p_{i} v_{j}
\end{gathered}
$$

where, $h_{i}, j_{i}, k_{i}$ are components of vectors called h-connection vector and $u_{i}, v_{i}, w_{i}$ are called components of v-connection vector respectively.

The equation (1.2.10) and (1.2.11) may be written as

$$
\begin{aligned}
& \mathrm{e}_{\alpha)(j}^{\mathrm{i}}=\mathrm{H}_{\alpha) \beta \gamma} \mathrm{e}_{\beta)}^{\mathrm{i}} \mathrm{e}_{\gamma) j} \\
& \left.\mathrm{e}_{\alpha)}^{\mathrm{i}}\right|_{\mathrm{j}}=\mathrm{V}_{\alpha) \beta \gamma} \mathrm{e}_{\beta)} \mathrm{e}_{\gamma) \mathrm{j}}
\end{aligned}
$$

The surviving scalar components ofH $\mathrm{f}_{\alpha \gamma \gamma}$ and $\mathrm{V}_{\alpha) \beta \gamma}$ are given by

$\mathrm{V}_{1) 1 \gamma}=\mathrm{V}_{2) 2 \gamma}=\mathrm{V}_{3) 3 \gamma}=\mathrm{V}_{4) 4 \gamma}=0, \quad \mathrm{~V}_{2) 1 \gamma}=\mathrm{V}_{1) 2 \gamma}=-\delta_{2 \gamma}, \mathrm{V}_{2) 3 \gamma}=-\mathrm{V}_{3) 2 \gamma}=\mathrm{u}_{\gamma}, \mathrm{V}_{2) 4 \gamma}=-\mathrm{V}_{4) 2 \gamma}=\mathrm{v}_{\gamma}$, $\mathrm{V}_{3) 4 \gamma}=-\mathrm{V}_{4) 3 \gamma}=\mathrm{w}_{\gamma}, \quad \mathrm{V}_{3) 1 \gamma}=-\mathrm{V}_{1) 3 \gamma}=-\delta_{3 \gamma}, \mathrm{V}_{4) 1 \gamma}=-\mathrm{V}_{1) 4 \gamma}=-\delta_{4 \gamma}, \quad \mathrm{H}_{2) 3 \beta}=\mathrm{h}_{\beta}=-\mathrm{H}_{3) 2 \beta}, \quad \mathrm{H}_{4) 2 \beta}=$ $\mathrm{j}_{\beta}=-\mathrm{H}_{2) 4 \beta}, \quad \mathrm{H}_{3) 4 \beta}=\mathrm{k}_{\beta}=-\mathrm{H}_{4) 3 \beta}$.

where, $\left.\mathrm{h}_{\alpha}, \mathrm{j}_{\alpha^{\prime}} \mathrm{k}_{\alpha}\right)$ and $\left(\mathrm{u}_{\alpha}, \mathrm{v}_{\alpha}, \mathrm{w}_{\alpha}\right)$ ( are scalar components ofh $\mathrm{i}_{\mathrm{i}}$ and $\mathrm{v}_{\mathrm{i}}$ respectively $\mathrm{h}_{\alpha}=\mathrm{h}_{\mathrm{i}} \mathrm{e}_{\alpha)}^{\mathrm{i}}, \quad \mathrm{j}_{\alpha}=\mathrm{j}_{\mathrm{i}} \mathrm{e}_{\alpha}^{\mathrm{i}}$, $\mathrm{k}_{\alpha}=\mathrm{k}_{\mathrm{i}} \mathrm{e}_{\alpha}^{\mathrm{i}}, \quad \mathrm{v}_{\alpha}=\mathrm{v}_{\mathrm{i}} \mathrm{e}_{\alpha)}^{\mathrm{i}}, \quad \mathrm{u}_{\alpha}=\mathrm{u}_{\mathrm{i}} \mathrm{e}_{\alpha}^{\mathrm{i}}, \mathrm{w}_{\alpha}=\mathrm{w}_{\mathrm{i}} \mathrm{e}_{\alpha}^{\mathrm{i}}$. The first scalar component $\mathrm{v}_{1}=\mathrm{v}_{\mathrm{i}} \mathrm{l}^{\mathrm{i}}$ vanishes identically in $\mathrm{a}$ four-dimensional Finsler space.

The h-scalar derivative of the adopted components $T_{a \beta}$ of the tensor $\mathrm{T}_{\mathrm{j}}^{\mathrm{i}}$ of $(1,1)$ type is defined as

$$
\mathrm{T}_{\alpha \beta, \gamma}=\frac{\delta \mathrm{T}_{\alpha \beta}}{\delta \mathrm{x}^{\mathrm{k}}} \mathrm{e}_{\gamma)}^{\mathrm{k}}+\mathrm{T}_{\mu \beta} \mathrm{H}_{\mu) \alpha \gamma}+\mathrm{T}_{\alpha \mu} \mathrm{H}_{\mu) \beta \gamma}
$$

where $\frac{\delta}{\delta \mathrm{x}^{\mathrm{k}}}=\frac{\partial}{\partial \mathrm{x}^{\mathrm{k}}}-\mathrm{G}_{\mathrm{k}}^{\mathrm{r}} \frac{\partial}{\partial \mathrm{y}^{\mathrm{r}}}$ and $\mathrm{G}_{\mathrm{k}}^{\mathrm{r}}$ are non-linear connection of $C \Gamma$.

Similarly the v-scalar derivative of the adopted components $\mathrm{T}_{\alpha \beta}$ of $T$ is defined as,

$$
\mathrm{T}_{\alpha \beta ; \gamma}=\mathrm{L} \frac{\partial \mathrm{T}_{\alpha \beta}}{\partial \mathrm{y}^{\gamma}} \mathrm{e}_{\gamma)}^{\mathrm{k}}+\mathrm{T}_{\mu \beta} \mathrm{V}_{\mu) \alpha \gamma}+\mathrm{T}_{\alpha \mu} \mathrm{V}_{\mu) \beta \gamma}
$$

Thus $\mathrm{T}_{\alpha \beta, \gamma}$ and $\mathrm{T}_{\alpha \beta ; \gamma}$ are adopted components of $\mathrm{T}_{\mathrm{j} \mid \mathrm{k}}^{\mathrm{i}}$ and $\mathrm{L} \mathrm{T}_{\mathrm{j}}^{\mathrm{i}} \mathrm{l}_{\mathrm{k}}$ respectively. i. e.

$$
\begin{array}{r}
\mathrm{T}_{\mathrm{j} \mid \mathrm{k}}^{\mathrm{i}}=\mathrm{T}_{\alpha \beta \gamma \gamma} \mathrm{e}_{\alpha)}^{\mathrm{i}} \mathrm{e}_{\beta) \mathrm{j}} \mathrm{e}_{\gamma) \mathrm{k}} \\
\left.\mathrm{LT}_{\mathrm{j}}^{\mathrm{i}}\right|_{\mathrm{k}}=\mathrm{T}_{\alpha \beta ; \gamma}\left(\mathrm{e}_{\alpha)}^{\mathrm{i}} \mathrm{e}_{\beta) \mathrm{j}} \mathrm{e}_{\gamma) \mathrm{k}}\right.
\end{array}
$$

\section{Geodesics}

The curve for shortest length, measured along the surface between any two points on the surface is called geodesic curve or geodesic.

\section{Geodesic Equation from Geodesic Curve with Finsler Space}

We know that

$$
d s=\sqrt{-g_{\alpha \beta} \frac{d x^{\alpha}}{d s} \frac{d x^{\beta}}{d s}} d s
$$


To extremize length take $\delta \int d s=0$

$$
\Rightarrow \int d s=\int\left(-g_{\alpha \beta} \frac{d x^{\alpha}}{d s} \frac{d x^{\beta}}{d s}\right)^{1 / 2} d s
$$

According to the Euler's - Lagrange equation

$$
\begin{aligned}
& \frac{d}{d s}\left(g_{\alpha \beta} u^{\alpha}\right)=\frac{1}{2} g_{\alpha \gamma, \beta} u^{\alpha} u^{\gamma} \\
& \text { Where }\left(-g_{\alpha \beta} \frac{d x^{\alpha}}{d s} \frac{d x^{\beta}}{d s}\right)=1 \text { and } u^{\alpha}=\frac{d x^{\alpha}}{d s} \\
& \text { Hence } \frac{d}{d s}\left(g_{\alpha \beta u^{\alpha}}\right)=g_{\alpha \beta} \frac{d u^{\alpha}}{d s}+g_{\alpha \gamma, \beta} u^{\alpha} u^{\gamma}
\end{aligned}
$$

Hence equation (2.1) becomes

$$
g_{\alpha \beta} \frac{d^{2} x^{\alpha}}{d s^{2}}+u^{\alpha} u^{\gamma}\left(g_{\alpha \beta, \gamma}-\frac{1}{2} g_{\alpha, \beta}\right)=0
$$

Now we use

And multiply equation (2.3) by $g^{\beta /}$ to obtain

$$
u^{\alpha} u^{\gamma} g_{\alpha \beta, \gamma}=u^{\alpha} u^{\gamma} \frac{1}{2}\left(g_{\alpha \beta, \gamma}+g_{\not \beta, \alpha}\right)
$$

$$
\frac{d^{2} x^{\tau}}{d s^{2}}+\frac{1}{2} g^{\beta \gamma}\left(g_{\alpha \beta, \gamma}+g_{\not \beta, \alpha}-g_{\alpha \gamma, \beta}\right) u^{\alpha} u^{\gamma}=\frac{d^{2} x^{\tau}}{d s^{2}}+\Gamma_{a \gamma}^{\tau} \frac{d x^{\alpha}}{d s} \frac{d x^{\gamma}}{d s}=0
$$

Since a co-ordinate form

$$
I_{\nu \lambda}^{\mu}=\frac{l}{2}\left(g_{\alpha v, \lambda}+g_{\alpha \lambda, v-g_{v \lambda \alpha}}\right) g^{\mu \alpha}
$$

Again from (1.1.1) \&(1.1.9) We have

$$
\begin{gathered}
\Rightarrow \Gamma_{\alpha \gamma}^{\tau}=\frac{1}{2}\left(g_{\beta \alpha, \gamma}+g_{\beta \gamma, \alpha}-g_{\alpha \gamma, \beta}\right) g^{\tau \beta} \\
u^{\alpha}=\frac{d x^{\alpha}}{d s}, \quad u^{\gamma}=\frac{d x^{\gamma}}{d s}
\end{gathered}
$$

From geodesics integral

$$
d s=L\left(x^{i}, d x^{i}\right)=\left[g_{i j}\left(x^{k}\right) d x^{i} d x^{j}\right]^{\frac{l}{2}}
$$

$$
g_{\alpha \beta}=-g_{i j}\left(x^{k}\right) \frac{d x^{i}}{d x^{\alpha}} \frac{d x^{j}}{d x^{\beta}}
$$

Again from (2.2) we have

$$
\begin{aligned}
& g_{\alpha \beta, \gamma}=\left[\frac{d}{d s}\left(g_{\alpha \beta} u^{\alpha}\right)-g_{\alpha \beta} \frac{d u^{\alpha}}{d s}\right] \frac{1}{u^{\gamma} u^{\alpha}} \\
& g_{\gamma \beta, \alpha}=\left[\frac{d}{d s}\left(g_{\gamma \beta} u^{\gamma}\right)-g_{\gamma \beta} \frac{d u^{\gamma}}{d s}\right] \frac{1}{u^{\alpha} u^{\gamma}} \\
& g_{\alpha \gamma, \beta}=\left[\frac{d}{d s}\left(g_{\alpha \gamma} u^{\alpha}\right)-g_{\alpha \gamma} \frac{d u^{\alpha}}{d s}\right] \frac{1}{u^{\alpha} u^{\beta}}
\end{aligned}
$$

From (2.6),(2.7),(2.8)\&(2.9) we have the geodesics equation of the form

\section{Theorem-}

$$
\frac{d^{2} x^{\tau}}{d s^{2}}+\frac{1}{2} g^{\beta \gamma}\left[g_{i k}\left(x^{j}\right) \frac{d x^{i}}{d x^{\alpha}} \frac{d x^{k}}{d x^{\gamma}}-g_{i j}\left(x^{k}\right) \frac{d x^{i}}{d x^{\alpha}} \frac{d x^{j}}{d x^{\beta}}-g_{k j}\left(x^{i}\right) \frac{d x^{k}}{d x^{y}} \frac{d x^{j}}{d x^{\beta}}\right]=0
$$

The geodesic of the velocity space metric defined in $d s^{2}=d \chi^{2}+\sinh ^{2} \chi\left(d \theta^{2}+\sin ^{2} \theta d \emptyset^{2}\right)$.

where the magnitude of the velocity is $\mathrm{v}=\tanh \chi$ are paths of minimum fuel for a rocket ship changing its velocity.

Proof -the geodesic is the path between two velocities which minimizes the arc -length between them, but arc length in the velocity space is just the magnitude of a small change of velocity. Science a rocket expends fuel monotonically for the boost it requires the geodesic of velocity space are paths of minimum fuel use.

\section{References}

[1]. Berwald, L, (1947):Uber Finslersche und Cartansche Geometries IV. Projective Krummungallgemeneraffiner Raume and Finselersche Raume skalarer Krummunrg, Ann. of Math. (2), 48, 755-781

[2]. Cartan, E(1934).:Les Espaces de Finsler. Actualities ScientifiquesIndustrilles no.79, Paris, Hermann .

[3]. Finsler, P. (1918):UberKurven and Flachen in allgemeinen Dissertation, Gottingen,

[4]. Hombu, H.(1934):KonformeInvariatenimFinslerschen Raume, J. Fac. Sci.Hokkaido Univ.12, 157-168

[5]. H. Rund(1959).; The differential geometry of finsler spaces, spcingerKerlag.

[6]. Matsumoto, M. and Miron R. (1977) :On an invariant theory of The Finsler Spaces, Period.Math.Hungary, 8,73-82.

[7]. Matsumoto, M. and Shimada, H.(1977) :On Finsler spaces with the curvature

[8]. tensorPhijk and Shijk satisfying special conditions. Reports on Mathematical

[9]. Physics. 12, 17-87.

[10]. Matsumoto, M. and Numata, S.(1979):On Finsler spaces with cubic metric, Tensor, N. S. 33, 153-162.

[11]. Matsumoto, M.(1986): Foundations of Finsler geometry and special Finsler spaces, Kaiseisha Press, Saikawa, Otsu, Japan,

[12]. Miron, R.( 1988 ):The geometry of higher-order Finsler spaces. Hadronic Press, Inn. USA, (ISBN 1-57485-033-4).

[13]. Matsumoto, M. (1989) :A slope of a mountain is a Finsler surface with respect to a time measure, J.math. Kyoto Univ. 29, 17-2. 
[14]. Pandey, T.N.and Diwedi, D.K. (1997) : A theory of Four dimensional Finsler spaces in terms of scalars, J. Nat. Acad. Math., 11, 176-190.

[15]. Pandey, T. N., Chaubey, V. K. and Prasad, B(2008). N.:Scalar Curvature of Two-dimensional Cubic Finsler spaces, Jour. International Acad. Of Phys. Sciences, Vol. 12, 127-137.

[16]. Pandey, T. N. and Chaubey, V. K. (2011) :Theory of Finlser spaces with $(\gamma, \beta)$-metric, Bulletin of the Transilvania University of Brasov, Vol. 4(53), No. 2, 43-56.

[17]. Randers,G.(1941): On an asymmetric metric in the four-space of general relativity, Phy. Rev. (2)59, $195-199$.

[18]. Wegener,J.M.(1935):UntersuchungderzwedreidimensionalenFinslerschenRaumemit der Grundform $\mathrm{L}=\left(\mathrm{a}_{\mathrm{ikl}} \mathrm{X}^{\mathrm{i}} \mathrm{X}^{\mathrm{k}} \mathbf{X}^{l}\right)^{1 / 3}$, Akad. Wetensch. Proc., 38, 949-955.

[19]. Wagner, V.V.(1938): Two-dimensional space with the metric defined by a cubic differential form, (Russian and English), Abh. Tscherny, Staatuniv. Saratow, 1, 29-40.

[20]. Wagner, V. V.(1943): On generalized Berwald spaces, C. R. Dokl. Acad. Sci., URSS, N.S., 39, 3 -5. 